\title{
Upsampling Based Back Projection Imaging algorithm for Multi-receiver Synthetic Aperture Sonar
}

\author{
Xianhua Wang, Xuebo Zhang, Sanwen Zhu
}

Sci. \& Tech. on Underwater Acoustic Antaganizing Laboratory, Zhanjiang 524022, P. R. China

\author{
Xianhua_w@163.com
}

Keywords: synthetic aperture sonar, multi-receiver, zero-padding in frequency domain, back projection algorithm, range migration correction.

\begin{abstract}
In order to solve problems including the interpolation error, the distortion of phase information and the huge computational costs by using classical truncated-interpolation based back projection (BP) algorithm for multi-receiver synthetic aperture sonar (SAS), a novel BP algorithm is proposed by exploiting zero-padding in frequency domain, which is equivalent to interpolation in time domain. The presented algorithm firstly implements Fourier transform to echoed signal of each receiver, and then does the range cell migration correction (RCMC) considering the influence of the non "stop-and-hop" for each pixel in imaging scenario based on zero-padding in frequency domain and rounding in time domain. Finally, simulation and lake trial results further validate the performance of the proposed method.
\end{abstract}

\section{Introduction}

Synthetic aperture sonar (SAS) systems ${ }^{[1-2]}$ provide high resolution reflectivity maps of the imaging scenario by coherently combining the echoes collected along a large virtual array formed by a short real element mounted on a remote operated vehicle (ROV) or an autonomous underwater vehicle (AUV) moving along an almost rectilinear trajectory. Typical operational SAS systems are formed by a single transmitter and a short linear real array of receiving hydrophones ${ }^{[3-4]}$ both mounted on an underwater platform that can be either towed or automonously moving along a nominal linear trajectory. The imaportant step with SAS or interferometric synthetic aperture sonar (InSAS) ${ }^{[5-6]}$ is synthetic aperture image reconstruction, which is an inverse problem where the goal is to create an image of the seafloor reflectivity from measurements of the echoed signals along the synthetic aperture. Back projection (BP) ${ }^{[7-8]}$ is a reconstruction technique used widely in the medical imaging files [Callow] for the similar reconstruction problems encountered in computed tomography. BP algorithm takes the received signal for a given pulse and back-project that signal over a spherical arc to all the possible contributing image points. Once back projection is performed on the remainder of the pulses and accumulated, an estimate of the image is obtained. This method is an exact inversion technique. It works in both near and far field. Therefore, BP algorithm is also called optimum imaging method, which is often served as a reference to evaluate the performances of other imaging methods. Besides, BP algorithm copes with all array gemotries and arbitrary platform histories; even wobbly, circular, and spiral paths all with sway, surge, roll, and pitch.

The point scatter response (PSR) ${ }^{[9]}$, which follws the range migration trajectory, is space-variant. It makes SAS processing a nonseparable two-dimensional problem. The range to the different contributions is important for finding the focusing delays. Calculating these is essentially what the algorithm is all about. To implement this mehtod in practice, the available discrete fast-time samples of matched-filtered signal must be interpolated to recover corresponding value. However, the interpolator corresponding to the low-pass filter is infinite. It means that truncated-interpolator would lead to a loss of high resolution information over the entire image. This is unfavourable for the latter interferometric signal processing.

Based on the fact that zero-padding in frequency domain is equivalent to interpolate in time domain, this paper proposes a novel BP algorithm by exploiting zero-padding in frequency domain. The presented method needs caculation of accurate range history considering the influence of the 
non "stop-and-hop" ${ }^{[10]}$ for each pixel in imaging scenario. Then, the upsampling ration in fast-time domain is caculated according to the largest range migration cell. Meanwhile, the largest range error between upsampling data and the accurate range history must be controlled within $\lambda / 8$. After that, the range cell migration can be corrected by rounding method. Compared with conventional truncated-interpolation based BP algorithm, the presented method is phase preserving. Besides, the efficiency can also be improved due to FFTW, which is developped by Frigo and Johnson in MIT. Finally, simulation and lake trial results further validate the performance of the proposed method.

\section{SAS signal model of multi-receiver}

On the two-dimensional plane, define the direction of $r$ as the distance dimension and define the direction of $x$ as the azimuth dimension, which is also the direction of sonar platform. Two-dimensional imaging geometry for multi-receiver SAS is shown in Fig. 1.

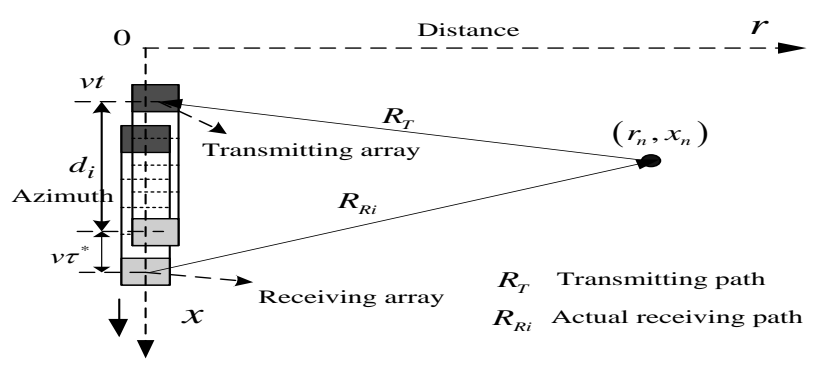

Fig. 1 Two-dimensional imaging geometry for multi-receiver SAS

As the sonar platform moves forward at velocity $v$, the transmitting arrays transmit linear frequency-modulated signal with fixed pulse-recurrence-frequency to the side direction, which is irrelevant with platform position. The linear frequency-modulated signal can be written as

$$
p(\tau)=\operatorname{rect}\left(\tau / T_{p}\right) \exp \left\{j \pi \gamma \tau^{2}\right\} \cdot \exp \left\{j 2 \pi f_{c} \tau\right\}
$$

Where, $\operatorname{rect}(\cdot)$ is a threshold function; $T_{p}$ is the pulse width; $\gamma$ is modulation frequency; $f_{c}$ is carrier frequency; $\tau$ is the time at distance dimension.

Assume there exist $m$ targets in the area of image reconstruction, $\left(r_{n}, x_{n}\right)(n=1,2 \ldots, m)$, where $r_{n}$ is the coordinates of skewed distance; $x_{n}$ is the azimuth coordinates. Then the distances between the transmitting array at position $v \cdot t$ and $n$ point target, $R_{T_{-} n}$, can be written as

$$
R_{T_{-} n}(t)=\sqrt{\left(r-r_{n}\right)^{2}+\left(v t-x_{n}\right)^{2}}
$$

The application of multi-receiver arrays improves the imaging speed and imaging distance of the platform. Thus, during the period of signal propagation $\tau^{*}$, the receiving array, at a distance $d_{i}$ with the transmitting array has already moved along the direction dimension with a distance of $v \cdot \tau^{*}$. Then the actual propagation distance $R_{R_{-} n}^{i}$ between the receiving array and $n^{\text {th }}$ point target can be written as

$$
R_{R_{-} n}^{i}(t)=\sqrt{\left(r-r_{n}\right)^{2}+\left(v t+v \tau^{*}+d_{i}-x_{n}\right)^{2}}
$$

Where the superscript $i$ stands for the $i^{\text {th }}$ receiving array.

Thus, the echo signal received by the $i^{\text {th }}$ receiving array is

$$
S S_{i}(\tau, t)=\sum_{n} p\left(\tau-\tau_{n}^{i}\right)
$$

Where $\tau_{n}^{i}=\left(R_{T_{-} n}+R_{R_{-} n}^{i}\right) / c$ is the two-way propagation delay the $n^{\text {th }}$ target, determined by $R_{T_{-} n}$ and $R_{R_{-}}^{i}$. 


\section{Back projection algorithm}

By adopting the coherence property of signals, BP imaging algorithm coherently sums along the expansion function in the time-domain corresponding to a certain pixel point. Consequently, the echoed signal with the same phase increases, and that with different phases attenuates. The diagram of BP algorithm is shown in Fig. 2. As the differences between the time delays of scatter point at different azimuths are not necessarily integers, in order to avoid its influence on imaging accuracy, interpolation is often applied to obtain these differences. However, the interpolator corresponding to the low-pass filter is infinite. It means that truncated-interpolator would lead to a loss of high resolution information over the entire image. This is unfavourable for the latter interferometric signal processing.

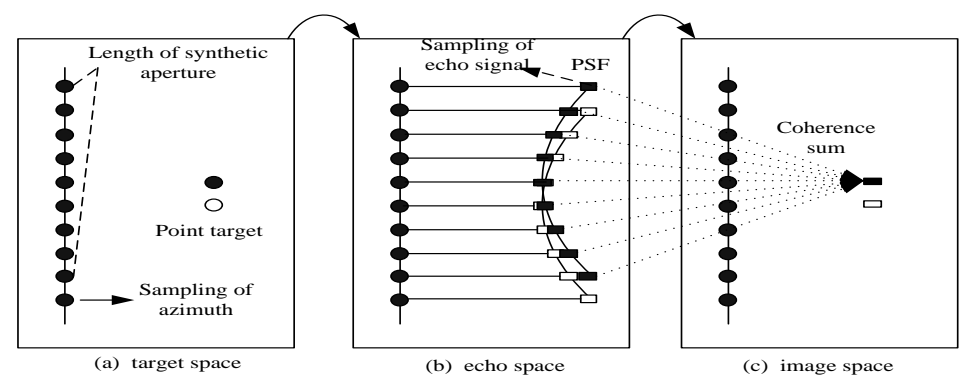

Fig. 2 Diagram of BP algorithm

As time delays in time domain correspond to frequency shift in frequency domain, a frequency-shifting based BP algorithm is presented. Its processing procedures are as follows.

1) Pulse compression in range

The pulse compressed signal is derived through match filtering in range for the received sigal of all arrays.

$$
s s_{p}(\tau, t)=s s(\tau, t) \otimes p^{*}(-\tau)
$$

Where $p^{*}(-\tau)$ denotes the complex replica of the transmitted signal. As shown in Fig.2(b), the energy distribution of the pulse compressed signal in invariant in azimuth. This step is implemented in frequency domain.

2) range migration correction

Range migration is corrected through zero-padding in frequency domain while it is implemented throuth truncating interpolation along the range migration curve in traditional pixel-by-pixel algorithm. First, the maximal range migration in synthetic aperture length Ls to the farest target is calculated to determine interpolating multiple in frequecy domain. Second, the pulse-compressed echo signal is zero-padded in frequency domain and transformed into time domain.

First, synthetic aperture length Ls to the point target $\left(r_{n}, x_{n}\right)$ in the scene is calculated, and the nearest distance between the point target $\left(r_{n}, x_{n}\right)$ and the platform moving direction is determined. Then, accurate time delay $\Delta \tau_{n}^{i}$ between each sampling location and the point target $\left(r_{n}, x_{n}\right)$ with reference to the nearest distance is calculated in turn. Finally, range migration and phase compensation is performed based on the relationship between the time delay $\Delta \tau_{n}^{i}$ and the resampling time in range.

$$
s s^{i}\left(\tau_{n}, t_{n}\right)=s s^{i}\left(\tau-\text { round }\left[\text { Interp } \cdot \Delta \tau_{n}^{i} / T\right]_{s}, t\right) \cdot \exp \left\{j 2 \pi f_{c} \Delta \tau_{n}^{i}\right\}
$$

Where $\left(\tau_{n}, t_{n}\right)$ is the time relative to $\left(r_{n}, x_{n}\right)$, and round [.] denotes round operation, and $T_{s}$ is the original sampling interval. In Eq. 6, envelope is shifted in the first item and azimuth doppler is compensated in second item.

Range migration to targets of the reference range is corrected accurately by the above processsing. As show in Fig.2(b), a point in the range migraton curve is corrected to a point in a strait line with 
IFFT transform in Eq. 6. For all points in the range migration curve, the range migration curve can be corrected to a strait line by performing the same operation.

3) Coherent summation in azimuth

Range migration curve to point targe $\left(r_{n}, x_{n}\right)$ is accurately decoupled in two dimensions by the operation in step 2. Therefore, coherent summation in a synthetic aperture is performed to the signal processed in step 2, and the image of the target can be obtained.

$$
f f\left(r_{n}, x_{n}\right)=\sum_{t \in\left[-L_{s} / 2 v, L_{s} / 2 v\right]} s s^{i}\left(\tau_{n}, t_{n}\right)
$$

4) Repeat the processing of step 2) and step 3) for all pixels and the imaging result will be obtained for the whole scene.

The method is easy to operate, requiring only Fourier Transform and cpmplex multiplication. Although zero-padding in frequency domain increases data length, if FFTW algorithm, developed by M. Frigo and S. Johnson of MIT, is adopted in Fourier inverse tranform, the influence of the increase of data length on the calculation amount is not significant. In addition, considering the rapid development of GPU and concurrent computation, if the proper optimization is adopted for the program, the computation efficiency will be promoted further. The method considers both computation efficiency and imaging precision. In combination the advantage of convenience in motion compensation, it will play an important role in the future.

\section{Result of Simulation Experiment and Lake Test}

Result of simulation Experiment. In order to validate the effectiveness, a simulation test is designed. The parameters of simulation are set up as follows. central frequency $150 \mathrm{kHz}$; band width of LFM signal is $20 \mathrm{kHz}$; pulse period is $0.2 \mathrm{~s}$, towed speed of the platform is $2.5 \mathrm{~m} / \mathrm{s}$; the size of transmitting array at the azimuth dimension is $0.08 \mathrm{~m}$; the size of receiving array at the azimuth dimension is $0.04 \mathrm{~m}$; the number of receiving arrays is 25 . The two-dimensional coordinates of simulated point target is $(74 \mathrm{~m}, 6 \mathrm{~m})$. Firstly, non-interpolation method (time-shift based on half-rounding method) and truncated interpolation method are used for simulated data to correct range migration. The imaging processing results are respectively shown in Fig. 3(a) and Fig. 3(b), while the imaging result of the algorithm presented here is shown in Fig. 3(c).

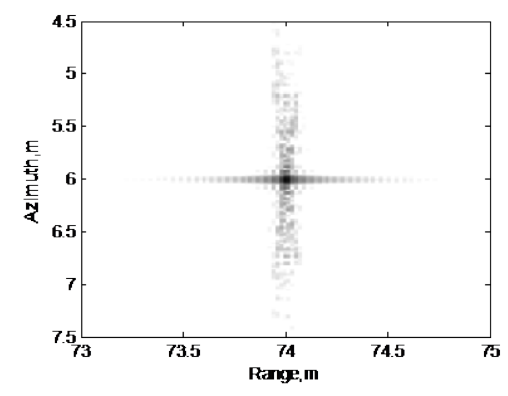

(a)Imaging result with non-interpolation method.

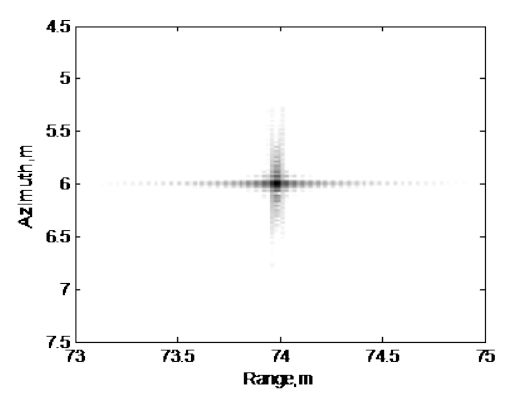

(b)Imaging result with interpolated method

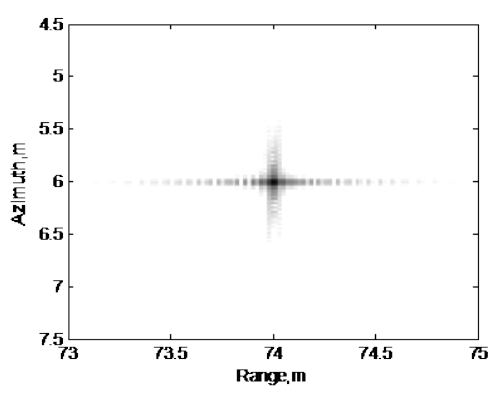

(c)Imaging result with the proposed method

Fig. 3 Imaging result of ideal targets 
In order to demonstrate more directly the imaging result, the azimuth slices of the point target in scene centre for the above-mentioned three methods are given in Fig. 4.

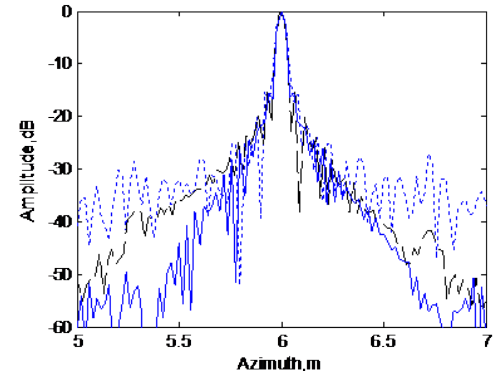

(a) Azimuth slice

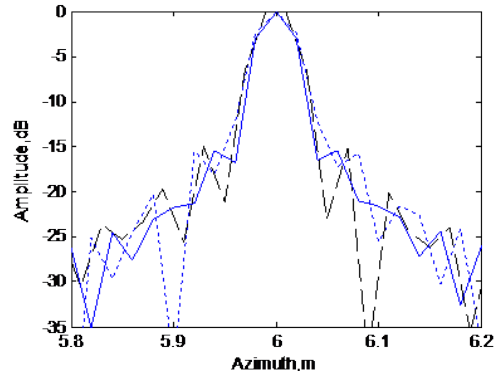

(b) Azimuth slice around the main lobe

Fig.4 Azimuth slice for the target in scene centre.

Compared the imaging results of the three methods, it is easy to see that the method presented here has improved the quality of target focusing and obtained better imaging result.

\section{Result of Lake Test}

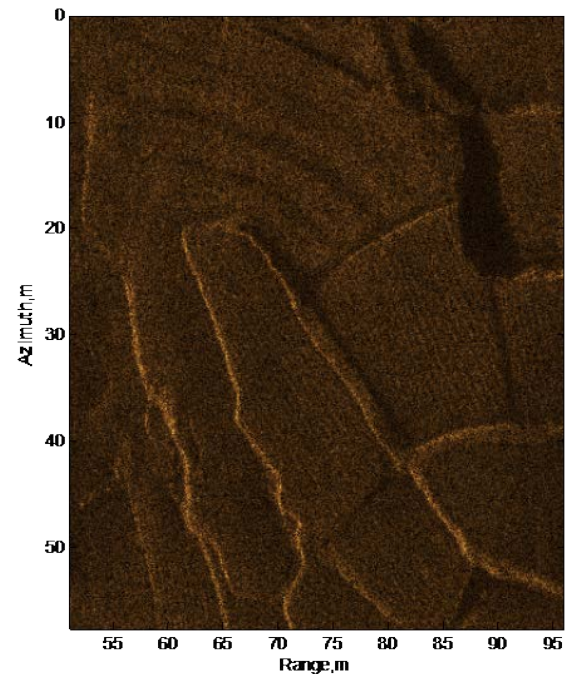

(a)Imaging result with non-interpolated method

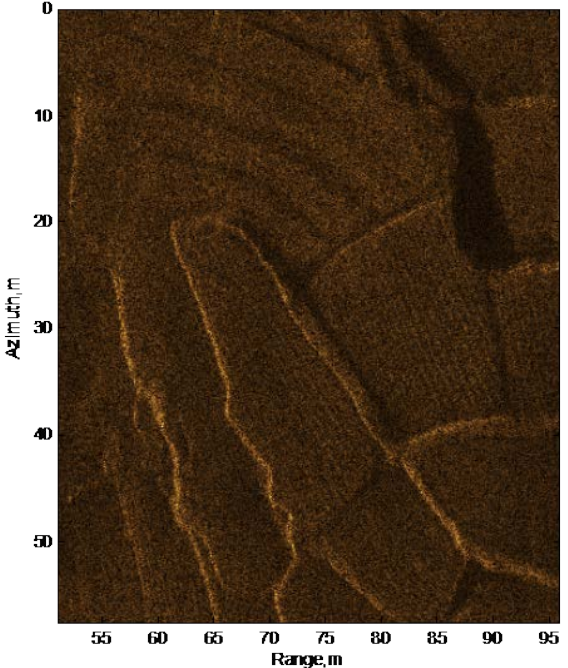

(b)Imaging result with interpolated method

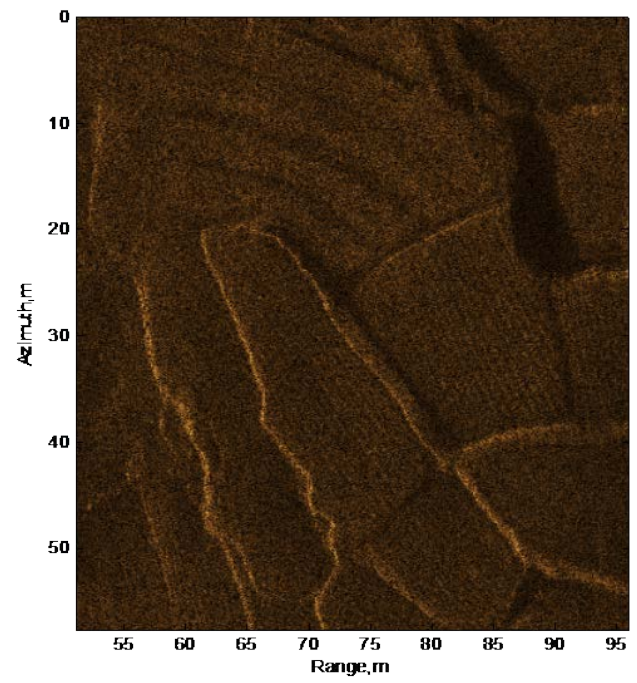

(c)Imaging result with proposed method

Fig. 6 Imaging result of lake trial

In 2010, a lake test is made in Thousand-islet Lake for product ChinSAS-150. The recorded data in the lake test is used to further demonstrate the validity and reliability of the presented method. 
The central frequency is $150 \mathrm{kHz}$; band width of LFM signal is $20 \mathrm{kHz}$; towed speed of the platform is $2.5 \mathrm{~m} / \mathrm{s}$; the aperture of each array is $0.04 \mathrm{~m}$. Before data processing, the data from each receiving arrays are sumed in the following way, $1+2+3 \cdots$. The imaging results of non-interpolation method, interpolation method and the presented method here are respectively shown in Fig. 5(a), Fig. 5(b) and Fig. 5(c). From comparison, it can be seen that the focusing results of Fig. 5(c) is finer, showing the validity of the method presentd here.

\section{Conclusion}

Back projection algorithm is a precise imaging algorithm in theory. However, due to the infinite interpolator,it is necessary to use the truncated interpolation in engineering to correct range migration, leading to the loss of phase information, which is of great importance in coherence signal processing. The method presented here adopts the precise time delays to precisedly correct range migration, avoiding the problem resulting from traditional truncated inperpolation. The method not only considers the imaging accuracy, but also the computational efficiency.

\section{Reference}

[1] JIANG Nan, Sun Da-jun,TIAN Tan. Comparison of RD and w-k imaging algorithms in synthetic aperture sonar[J].Journal of Naval University of Engineering, 2002,14(2):58-61. (in Chinese)

[2] Hayes M P, Gough P T. Synthetic aperture sonar: a review of current status [J]. Journal of Oceanic Engineering, 2009, 34(3): 207-224.

[3] Gilmour G A. Synthetic aperture side-looking sonar system [J]. Journal of the Acoustical Society of America, 2009, 65: 557.

[4] Sheriff R W. Synthetic aperture beamforming with automatic phase compensation for high frequency sonars [C]// Proceedings of the Symposium on Autonomous Underwater Vehicle Technology. Washington DC,USA: IEEE,1992.

[5] CHEN Ming, TANG Jin-song. A method of estimating initial phase of InSAS[J].Journal of Naval University of Engineering, 2010,22(5):10-15. (in Chinese)

[6] BAI Sheng-xiang, TANG Jin-song, ZHANG Sen. Method of DEM reconstruction of InSAS based on improved coherence[J].Journal of Naval University of Engineering, 2012,24(6):16-19. (in Chinese)

[7] XU Jiang, TANG Jin-song, ZHANG Chun-hua. Multi-aperture synthetic aperture sonar imaging algorithm [J].Signal Processing, 2003,19(2): 157-160. (in Chinese)

[8] Callow H J. Signal processing for synthetic aperture sonar image enhancement [D]. New Zealand: University of Canterbury, 2003.

[9] Bamler R. A comparison of range-Doppler and wavenumber domain SAR focusing algorithms [J]. IEEE Transactions on Geoscience and Remote Sensing, 1992, 30(4): 706-713.

[10] YANG Hai-liang, ZHANG Sen, TANG Jin-song. Study on Simulation of multiple-receiver Synthetic Aperture Sonar Imagery Based on Wide Swath [J]. Journal of System Simulation, 2011, 23(7):1424-1428. (in Chinese) 